Research Article

\title{
Food Safety Practices and Associated Factors among Food Handlers of Fiche Town, North Shewa Zone, Ethiopia
}

\author{
Samuel Chane Teferi (iD, Israel Sebsibe, and Birhanu Adibaru \\ Salale University, Department of Biology, P.O. Box 245, Fiche, Ethiopia \\ Correspondence should be addressed to Samuel Chane Teferi; samuelchane19@gmail.com
}

Received 24 September 2021; Revised 16 November 2021; Accepted 1 December 2021; Published 17 December 2021

Academic Editor: Issam A. Al-Khatib

Copyright ( $\odot 2021$ Samuel Chane Teferi et al. This is an open access article distributed under the Creative Commons Attribution License, which permits unrestricted use, distribution, and reproduction in any medium, provided the original work is properly cited.

\begin{abstract}
Background. Foodborne diseases remain a major public health problem globally, but the problem is severe in developing countries like Ethiopia. The objective of this study was to assess food safety practices and associated factors among food handlers of Fiche town. Methods. A cross-sectional study was conducted among 422 food handlers working in food and drink establishments. The data were collected using a structured questionnaire and observational checklist. Data were entered and coded into SPSS for analysis. Multivariable logistic regression was used to identify the predictor variables associated with the practice of food handlers $(p<0.05)$. Result. $61.6 \%$ of food handlers knew the potential risk of contaminating food with dirty hands, and $70 \%$ washed hands with soap before working with food. $52.8 \%$ of food handlers covered the hair with restraints. $66.8 \%$ of food handlers used outer garments, and the majority of food handlers (71.1\%) had a trimmed fingernail. Two hundred thirteen (50.5\%) of food handlers had good food handling practices. Medical checkup $(\mathrm{AOR}=3.16$; 95\% CI 1.89, 5.26), sanitary inspection (AOR = 1.76; 95\% CI 1.16, 2.69), knowledge ( $\mathrm{AOR}=2.31 ; 95 \% \mathrm{CI} 1.53,3.48)$, service year ( $\mathrm{AOR}=3.11 ; 95 \% \mathrm{CI} 1.53,6.31$ ), and educational status $(\mathrm{AOR}=3.42,95 \% \mathrm{CI} 1.29,9.04)$ were found to be significantly associated with food handling practices. Conclusion. The food handlers should take various training concerning food hygiene and safety to enhance their knowledge and practice. Regular sanitary inspection of food and drink establishments is recommended.
\end{abstract}

\section{Introduction}

Foodborne diseases remain a major public health problem globally and are responsible for significant morbidity and mortality rates [1]. According to the World Health Organization (WHO), more than 600 million illnesses and 420,000 annual deaths worldwide are due to contaminated food, and about 33 million disability-adjusted life years are attributable to foodborne infections globally [2]. Foodborne diseases are severe in developing countries due to difficulties securing optimal hygienic food handling practices and poor sanitation [3, 4]. Lifestyle change and urbanization lead people to dine away from home more often, contributing to the unregulated opening of eating establishments with inadequate hygiene conditions [5]. Estimating the magnitude of foodborne disease is complicated because reliable statistics are unavailable in most countries due to poor or nonexistent reporting systems [6].
Approximately $10-20 \%$ of foodborne illnesses outbreaks are because of contamination by food handlers [7]. Like other developing countries, foodborne diseases are prevalent in Ethiopia; the country's annual incidence of foodborne illnesses ranged from 3.4 to $9.3 \%$, the median being $5.8 \%$ [8].

Microbiological contamination of food occurs in catering establishments due to poor personal hygiene practices, inappropriate storage temperature, and dirty food contact surfaces [9]. Food handlers may be infected by a wide range of enteropathogens and engaged in transmitting many infections to the public. These intestinal parasites and enteropathogenic bacteria transmit directly or indirectly through objects contaminated with feces [10-12]. Salmonella and Shigella are among the common causes of foodborne diseases throughout the world [13]. Food handlers are anyone who directly handle packaged or unpackaged food, food equipment, and utensils (such as cutlery, plates, bowls, or chopping boards) [14]. 
There are many factors associated with food handling practices. The major contributing factors for potential foodborne pathogen outbreaks in food and drink establishments are due to inadequate knowledge of food handlers [15-17], poor educational status [18], insanitary conditions of food and drink establishments $[17,19]$, and marital status $[16,20]$. Studies in different parts of Ethiopia showed poor food safety practices due to the factors mentioned above and weak regulatory systems, inadequate food safety laws, and lack of financial resources to invest in safer equipment in the country $[15,16,21]$.

According to Kibret and Abera [17] and Kumie and Zeru [22], good personal hygiene and food handling practices are the basis for preventing the transmission of pathogens from food handlers to consumers. Therefore, to reduce foodborne illnesses, it is crucial to understand the knowledge and practices of food handlers [23]. The number of food and drink establishments in Fiche town increases from time to time, and many people use the food and drink services in the city. Moreover, food safety practices and their associated factors among food handlers were unknown in the town. Hence, the present study aimed to assess food safety practices and associated factors among food handlers working in food and drink establishments in Fiche town. Since the study was cross-sectional, it will not show a cause and effect relationship, and the study might be liable to social desirability and recall bias.

\section{Methods}

2.1. Description of the Study Area. Fiche is a town in central Ethiopia. It is the North Shewa Zone of Oromia Region's administrative center and has four kebeles (Figure 1). It is located about $114 \mathrm{~km}$ north of Addis Ababa, the capital city of Ethiopia. Fiche has a latitude and longitude of $9^{\circ} 48^{\prime} \mathrm{N}$ and $38^{\circ} 44^{\prime} \mathrm{E}$, respectively. Fiche town has an elevation between $2,738 \mathrm{~m}$ and $2,782 \mathrm{~m}$ above the sea level.

2.2. Study Design. A community-based cross-sectional study was conducted to evaluate food handlers' practices and associated factors working in food and drink establishments in Fiche town.

2.3. Population and Sampling Procedures. Food handlers working in food and drink establishments such as hotels, café and restaurants, snack houses, restaurants, juice houses, and butcher shops in the study area were the focus of the study. The number of food handlers was challenging to estimate, according to the tourism office data. A simple random sampling technique was used to select food and drink establishments. Then, actively working food handlers were selected randomly. The sample size was determined using a single population proportion formula. By taking the proportion $(p)$ of $50 \%$, since there is no previous investigation in the town, significance level 5\% ( $\alpha=0.05), Z \alpha /$ $2=1.96$, the margin of error between the population and the sample $5 \%(d=0.05)$, and finally, $10 \%$ nonresponse rate was considered.

$$
\begin{aligned}
& n=\left(\frac{Z \alpha}{2}\right)^{2} P \frac{(1-P)}{d^{2}} \\
& n=(1.96)^{2} \times 0.5 \frac{(1-0.5)}{(0.05)^{2}}=384 .
\end{aligned}
$$

Therefore, the final sample size was 422 .

2.4. Data Collection Tools and Procedures. The data collection instrument used for this study includes a structured questionnaire and observational checklist adopted from related literature in Ethiopia and elsewhere. The questionnaire was first prepared in English, translated to local languages, and translated back to English to see any inconsistency. The pretest was performed on $5 \%$ of food handlers outside the sampling town; then, correction and modification were undertaken based on the gaps identified during the pretest. Data collectors and supervisors were oriented about the purpose of the study, the components of the questionnaire, and data quality management. The questionnaire was used to assess food handlers' sociodemographic characteristics (age, sex, educational status, marital status, service year, and income) and knowledge (cause, mode, and symptom of foodborne diseases). Observational checklists were used to assess food handlers' practice while they performed their task on-site. Respondents were assessed using 12 practicerelated checklists to evaluate the level of practice. Food handlers who scored less than or equal to the mean value $(\leq 50 \%)$ were considered as having a "poor level of practices," and those who scored greater than the mean value $(>58.3 \%)$ were considered as having a "good level of practices" $[16,17,21]$. Respondents were asked 8 knowledge-based questions to assess the level of knowledge. Respondents who scored less than or equal to the mean value $(\leq 50 \%)$ were considered as having a "poor level of knowledge," and those who scored greater than the mean value $(>62.5 \%)$ were considered as having a "good level of knowledge" $[16,17,24]$.

2.5. Study Variables. Food safety practice was the dependent variable in this study, and educational level, age, gender, work experience, medical checkup, marital status, income, knowledge, and sanitary inspection were the independent variables.

2.6. Data Analysis. Consistency and completeness of data were checked and verified during collection, entry, and analysis. Statistical Package for Social Sciences (SPSS) version 26 was used to analyze the data. Bivariate logistic regression was used to identify variables with $p$ value $<0.25$. Then, these variables were analyzed in multivariable logistic regression to assess the independent effect after controlling other variables [25]. The model fitness was checked by the Hosmer and Lemeshow goodness-of-fit test and was found to be 0.590 . Finally, a $p$ value less than 0.05 was considered for determining statistically significant variables. 


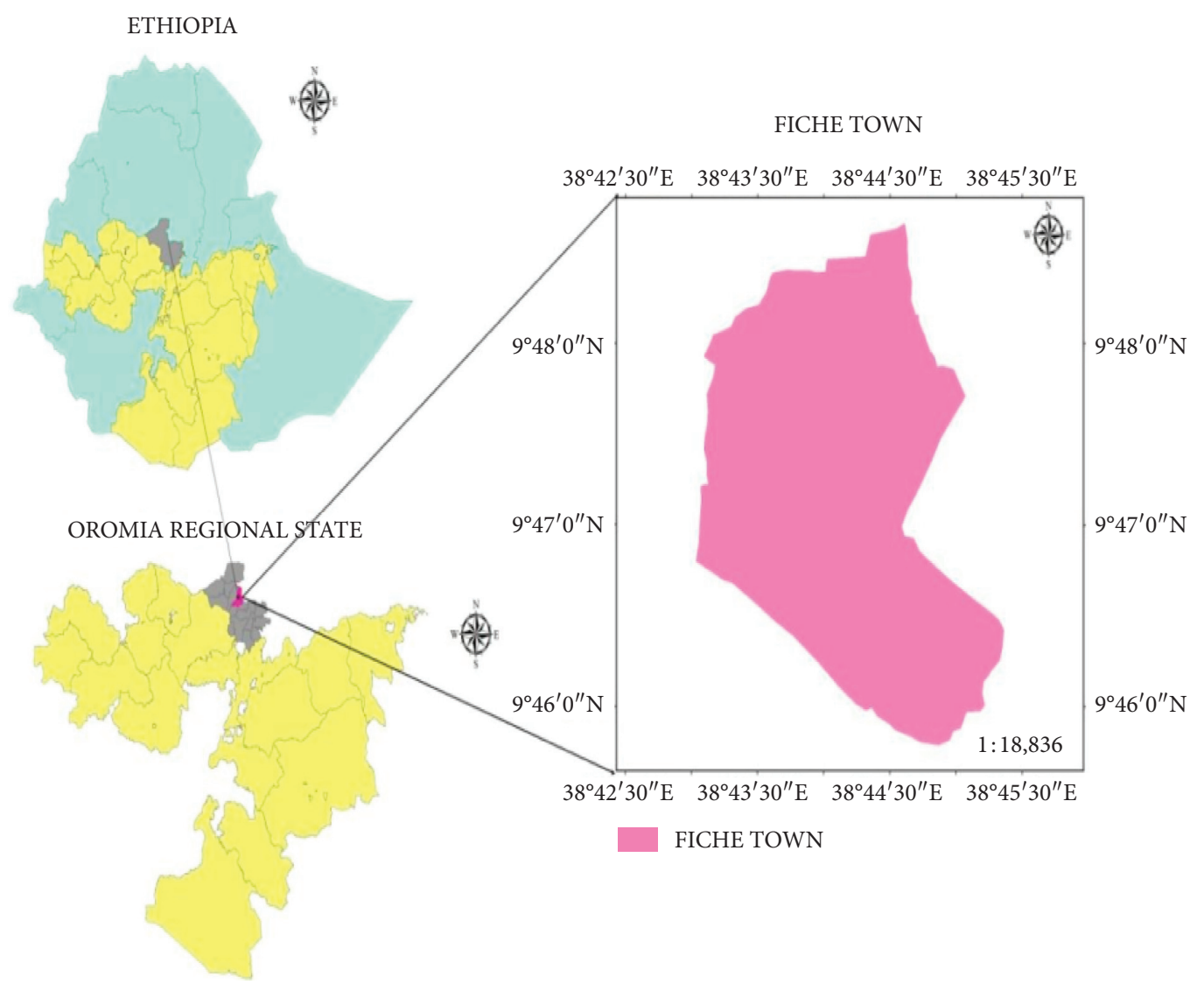

Figure 1: Map of the study area (source: ARC GIS database).

2.7. Ethical Considerations. The study was undertaken after approval by Salale University's research and community service directorate. During data collection, verbal and written informed consent was also obtained from study participants after the purpose of the study was explained. We kept the confidentiality of the respondents and for the food and drinking establishments by asking the participants not to write their names on the questionnaires and codes to hide the identity of the food and drinking establishments.

\section{Result}

3.1. Sociodemographic Characteristics of Food Handlers. The majority, 302 (71.6\%), of food handlers were females, and $120(28.4 \%)$ were males. $5.7 \%$ of respondents were illiterate. Most of the food handlers $(63.7 \%)$ were single, while $32.2 \%$ of them were married, and $199(47.2 \%)$ had an income of 1001-1500 Ethiopian birr (46 ETB=1 USD). Only 42 (9.9\%) food handlers had over ten years of service years (Table 1).

3.2. Knowledge of Food Handlers. The majority, 354(83.9\%), of food handlers had heard about foodborne diseases. Mass media (33.9\%) was the most common source of information, followed by friends and customers $(28.2 \%)$. The proportion of food handlers who believed that foodborne diseases are caused by contamination with bacteria and parasites was 280 $(66.4 \%)$, and $208(49.3 \%)$ responded that contaminated water was the channel for transmissions. Of those asked about the mode of transmission of foodborne disease, 248 $(58.8 \%)$ answered that contaminated food was the vehicle. $260(61.6 \%)$ of food handlers knew the potential risk of contaminating food with dirty hands (Table 2).

3.3. Practice of Food Handlers on Food Hygiene. Among the total food handlers observed during visits, $282(66.8 \%)$ used outer garments. The majority, 210 (74.5\%), of food handler's outer garments were clean. $223(52.8 \%)$ of food handlers covered hair with restraints. The majority of food handlers, $300(71.1 \%)$, had a trimmed fingernail.

3.4. Factors Associated with Food Handling Practice. Two hundred thirteen (50.5\%) food handlers had good food handling practices. Multivariable logistic regression analysis revealed that medical checkup $(\mathrm{AOR}=3.16$; 95\% $\mathrm{CI} 1.89$, 5.26), sanitary inspection $(\mathrm{AOR}=1.76 ; 95 \% \mathrm{CI} 1.16,2.69)$, knowledge $(\mathrm{AOR}=2.31 ; 95 \% \mathrm{CI} 1.53,3.48)$, service year $(\mathrm{AOR}=3.11 ; 95 \% \mathrm{CI} 1.53,6.31)$, and educational status (AOR $=3.42$; 95\% CI $1.29,9.04)$ were found to be significantly associated with food handling practices with $p$ value $<0.05$ (Table 3). 
TABLE 1: Sociodemographic characteristics of food handlers working in food and drink establishment in Fiche town $(n=422)$.

\begin{tabular}{lcc}
\hline Characteristics & Frequency & Percentage (\%) \\
\hline Sex & & \\
Male & 120 & 28.4 \\
Female & 302 & 71.6 \\
\hline Age & & \\
$\leq 20$ & 92 & 21.8 \\
$21-30$ & 274 & 64.9 \\
$31-40$ & 49 & 11.6 \\
$>40$ & 7 & 1.7 \\
\hline Educational status & & \\
Illiterate & 24 & 5.7 \\
Grades 1-6 & 125 & 29.6 \\
Grades 7-12 & 179 & 42.4 \\
Grades $>12$ & 94 & 22.3 \\
\hline Marital status & & \\
Single & 269 & 63.7 \\
Married & 136 & 32.2 \\
Divorced & 17 & 4 \\
\hline Service year of food handlers & \\
$\quad$ <1 year & 161 & 38.2 \\
1-5 years & 174 & 41.2 \\
6-10 years & 45 & 10.7 \\
$>10$ years & 42 & 9.9 \\
\hline Food handler's monthly income (in birr) & \\
500-1000 & 97 & 23 \\
1001-1500 & 199 & 29.8 \\
$>1500$ & 126 & \\
\hline
\end{tabular}

\section{Discussion}

Foodborne diseases remain a major public health problem across the globe. Still, the problem is severe in developing countries like Ethiopia due to difficulties securing optimal hygienic food handling practices [3]. Therefore, to reduce foodborne illnesses, it is crucial to understand the knowledge and practices of food handlers [23]. In this study, 213 (50.5\%) had good food safety practices from 422 food handlers, and $49.5 \%$ had poor food safety practices. Our finding is lower than studies conducted in Indonesia (90\%) [26], Bahir Dar (67.6\%) [27], Jordan (89.4\%) [28], Mekelle (63.9\%) [6], and Malaysia (59.3\%) [24]. The variation might be due to study settings and food safety culture. According to Nyarugwe et al. [29], taking food safety culture into account is a promising way to improve food safety performance in the food industry. However, the present finding was comparable with studies conducted in Debark town (40.1\%) [30], Dangila town (52.5\%) [16], Gondar town (49\%) [15], and Nigeria (50\%) [31]. The possible reason for discrepancies might be the difference in the study design, cutoff points, food handler's sociodemographic profile, and year of study.

Food handlers with a good level of knowledge were 2.31 times more likely to have good food safety practices than those with a poor level of knowledge ( $\mathrm{AOR}=2.31 ; 95 \% \mathrm{CI} 1.53,3.48)$. Our finding is supported by studies conducted in Gondar, Malaysia, Dangila, Mekelle, and Gondar town $[1,6,15,16,32]$. Having adequate knowledge regarding foodborne disease transmission mechanisms and ways of food contamination is
TABLE 2: Knowledge status of food handlers on food handling practices $(n=422)$.

\begin{tabular}{lcc}
\hline Variable & Frequency & $\begin{array}{c}\text { Percentage } \\
(\%)\end{array}$ \\
\hline Heard about foodborne disease & & \\
No & 68 & 16.1 \\
Yes & 354 & 83.9 \\
\hline If yes, your source of information $(n=354)$ & \\
Health center & 64 & 18.1 \\
Sanitarian during inspection & 70 & 19.8 \\
Mass media & 120 & 33.9 \\
Others (friends and customers) & 100 & 28.2 \\
\hline Cause of foodborne disease* & & \\
Contaminated with bacteria and & 280 & 66.4 \\
parasites & 90 & 21.3 \\
Adding chemicals & 2 & 0.5 \\
Anger of god & 250 & 59.2 \\
Unhygienic food preparation & 248 & \\
\hline Mode of transmission of foodborne disease* & 58.8 \\
Contaminated food & 208 & 49.3 \\
Contaminated water & 50 & 11.8 \\
Vectors (flies and cockroaches) & & \\
\hline Reason for food contamination* & 378 & 89.6 \\
Contact of unhygienic hands & 260 & 7.6 \\
Unhygienic working environment & 211 & 50 \\
Unclean utensils & 199 & 47.2 \\
Infected food handlers & 48 & 11.4 \\
Exposure to insects and rats & 6 & 1.4 \\
\hline Symptoms of foodborne disease* & & \\
Vomiting & 189 & 44.8 \\
Fever & 140 & 33.2 \\
Diarrhea & 277 & 65.6 \\
I do not know & 33 & 7.8 \\
\hline Germs found on cutting board & & \\
Yes & & \\
No & & \\
\hline Good personal hygiene prevents foodborne disease & \\
Yes & & \\
No & & \\
I do not know & & \\
\hline Becase of the possbity of & \\
\hline
\end{tabular}

${ }^{*}$ Because of the possibility of multiple responses, the total number of food handlers may not be equal to $422(100 \%)$.

essential to undertake particular basic practices to alleviate those problems [33]. But in the present study, only 149 (35.3\%) of food handlers had good knowledge of food safety. The present finding is higher than a study conducted in Dangila town (28.8\%) [16] but lower than a study conducted in Gondar (44.3\%) [15], India (58.3\%) [34], and Malaysia (51.6\%) [35]. This might be due to differences in the study setting, development of researched site, and sociodemographic profile of respondents. Food safety practice was significantly associated with sanitary inspection. The probability of having good food safety practice was 1.76 times higher among food handlers who were supervised than their counterparts $(\mathrm{AOR}=1.76 ; 95 \% \mathrm{CI}$ $1.16,2.69)$. The present finding was supported by a study conducted in Arba Minch and Gondar [15, 21]. This might be due to supervisors giving advice and feedback to food handlers, the owners, and the managers during an inspection. 
TABLE 3: Determinants of food safety practice among food handlers working in food and drinking establishments in Fiche town.

\begin{tabular}{|c|c|c|c|c|c|}
\hline \multirow[t]{2}{*}{ Variables } & \multicolumn{2}{|c|}{$\begin{array}{c}\text { Food safety } \\
\text { practice }\end{array}$} & \multirow[t]{2}{*}{ Wald } & \multirow[t]{2}{*}{ Sig. } & \multirow[t]{2}{*}{ AOR $(95 \% \mathrm{CI})$} \\
\hline & Good & Poor & & & \\
\hline \multicolumn{6}{|c|}{ Sanitary inspection } \\
\hline $\begin{array}{l}\text { Yes } \\
\text { No }\end{array}$ & $\begin{array}{c}161 \\
52\end{array}$ & $\begin{array}{c}133 \\
76\end{array}$ & $\begin{array}{c}7.05 \\
1\end{array}$ & $0.008^{*}$ & $1.76(1.16,2.69)$ \\
\hline \multicolumn{6}{|c|}{ Medical checkup } \\
\hline $\begin{array}{l}\text { Yes } \\
\text { No }\end{array}$ & $\begin{array}{c}64 \\
149 \\
\end{array}$ & $\begin{array}{c}25 \\
184 \\
\end{array}$ & $\begin{array}{c}19.54 \\
1 \\
\end{array}$ & $0.001^{*}$ & $3.16(1.89,5.26)$ \\
\hline \multicolumn{6}{|l|}{ Knowledge } \\
\hline $\begin{array}{l}\text { Good } \\
\text { Poor }\end{array}$ & $\begin{array}{c}95 \\
118 \\
\end{array}$ & $\begin{array}{c}54 \\
155 \\
\end{array}$ & $\begin{array}{c}15.95 \\
1 \\
\end{array}$ & $0.001^{*}$ & $2.31(1.53,3.48)$ \\
\hline \multicolumn{6}{|l|}{ Service year } \\
\hline$<1$ year & 59 & 102 & 1 & & \\
\hline $1-5$ years & 88 & 86 & 5.57 & $0.018^{*}$ & $0.27(0.09,0.80)$ \\
\hline $6-10$ years & 39 & 6 & 2.5 & 0.113 & $1.75(0.87,3.5)$ \\
\hline$>10$ years & 27 & 15 & 9.87 & $0.002^{*}$ & $3.11(1.53,6.31)$ \\
\hline \multicolumn{6}{|c|}{ Educational status } \\
\hline Illiterate & 7 & 17 & 1 & & \\
\hline $1-6$ & 53 & 72 & 0.35 & 0.552 & $1.16(0.70,1.93)$ \\
\hline $7-12$ & 98 & 81 & 5.52 & $0.019^{*}$ & $1.91(1.11,3.29)$ \\
\hline$>12$ & 55 & 39 & 6.17 & $0.013^{*}$ & $3.42(1.29,9.04)$ \\
\hline
\end{tabular}

*Statistically significant at $p<0.05$.

Food handlers who had medical checkups were 3.16 times more likely to have good food handling practices than those who had no medical checkups (AOR $=3.16$; $95 \%$ CI $1.89,5.26)$. This could be the healthcare workers who advised food handlers during the examination, enhancing their food handling practice. The present finding is supported by studies conducted in Arba Minch, Dessie, and Gondar town $[15,21,36]$. In the present study, service year (experience) was significantly associated with food safety practice. The odds of performing good food handling practice among food handlers who had $>10$ years' experience was 3.11 times higher than those who had less than one year experience $(\mathrm{AOR}=3.11 ; 95 \% \mathrm{CI} 1.53,6.31)$. In line with our finding, a study from Bahir Dar, Debark, and Gondar town reported that experienced staff had good food handling practice (AOR: 3.4; 95\% CI 1.8, 6.4; AOR: 1.95; 95\% CI 1.11, 3.45; AOR: 3.3703 ; 95\% CI 1.2943, 8.7846) [1, 27, 30]. The possible explanation might be food handlers acquired better knowledge and skills through repeated exposure. Similarly, the odds of performing good food handling practices among food handlers who have attained above grade twelve education were 3.42 times more likely to have good food handling practices than illiterates ( $\mathrm{AOR}=3.42 ; 95 \% \mathrm{CI} 1.29$, 9.04). The possible explanation might be the depth of knowledge could affect food handlers' food handling practices. The present finding is comparable with previous studies $[18,27,28,30]$. In the present study, food hygiene training was not significantly associated with food handling practice. In contrast to our study, other reports showed a significant association between training and food handling practices $[1,15,21,27]$. The possible reason might be that most food handlers did not take food hygiene training
$(80.3 \%)$ in this study. In agreement with our finding, a similar result was reported from Mekelle, Bahir Dar, and Dangila town $[5,16,17]$.

Of the 282 respondents who used outer garments in the present study, 210 (74.5\%) of the food handler's outer garments were clean. The present finding is comparable with a study conducted in Mekelle town (72.6\%) [22]. A higher result was reported in a study conducted in Bahir Dar (92.6\%) [17] and Dangila town (88.7\%) [16]. 223 (52.8\%) food handlers covered their hair with restraints in our study. The present study is comparable with a study conducted in Gondar town (51.5\%) [15]; however, it is much better than similar studies conducted in Zeway (40.1\%) [37] and Mekelle (39\%) [22].

Previous studies on handwashing compliance ranged $5 \%-50 \%$ among food handlers in food service facilities [38]. In line with previous investigations, 295 (70\%) food handlers wash hands with soap before working with food in our finding. A higher result was reported in South Africa, who found that $94 \%$ washed their hands under all circumstances [39]. Compared to other hand parts, the area beneath fingernails harbors most microorganisms and is the most difficult to clean [40]. Therefore, to prevent the transmission of foodborne diseases, food handlers working in food and drink establishments should trim their fingernails. In the present study, most food handlers 300 (71.1\%) had a trimmed fingernail. Our finding is supported by a study conducted in Ghana (63.3\%), Aligarh (69.9\%), and Mekelle town $(76.2 \%)[22,41,42]$.

\section{Conclusion and Recommendations}

The present study revealed that there was poor food handling practice among food handlers. The predominant factors associated with good food handling practices were medical checkups, educational status, sanitary inspection, knowledge, and service year. The food handlers should take various training concerning food hygiene and safety to enhance their knowledge and practice. Moreover, a regular sanitary inspection of food and drink establishments is recommended. Future studies should focus on the enumeration of bacteria from food utensils and food handlers.

\section{Abbreviations}

AOR: Adjusted odds ratio

ETB: Ethiopian birr

CI: Confidence interval

WHO: World Health Organization

SPSS: Statistical Package for Social Sciences.

\section{Data Availability}

The datasets used to support the findings of this study are available from the corresponding author upon request.

\section{Disclosure}

The funder (Salale University) did not take part in the design, data collection, analysis, and submission for publication of this study. 


\section{Conflicts of Interest}

The authors declare that they have no conflicts of interest.

\section{Acknowledgments}

The authors are grateful to all study participants, data collectors, and food and drink establishment owners/managers. This study was funded by Salale University.

\section{References}

[1] Z. Gizaw, M. Gebrehiwot, and Z. Teka, "Food safety practice and associated factors of food handlers working in substandard food establishments in gondar town, northwest Ethiopia, 2013/14," International Journal of Food Science, Nutrition and Dietetics, vol. 3, no. 7, pp. 138-146, 2014.

[2] World Health Organization (WHO), Food Safety-Key Facts, World Health Organization, Geneva, Switzerland, 2019.

[3] D. Grace, Food Safety in Developing Countries: An Overview, Evidence on Demand, London, UK, 2015.

[4] World Health Organization, WHO's First Ever Global Estimates of Foodborne Diseases Find Children under Account for Almost One Third of Deaths, World Health Organization, Geneva, Switzerland, 2015.

[5] World Health Organization, Global Strategy for Food Safety: Safer Food for Better Health, World Health Organization, Geneva, 2002.

[6] D. Nigusse and A. Kumie, "Food hygiene practice and prevalence of intestinal parasites among food handlers working in mekelle university student's cafeteria," Journal of Social Sciences, vol. 1, no. 4, pp. 65-71, 2012.

[7] N. Mudey, G. Kesharwani, A. Mudey, R. C. Goyal, A. K. Dawale, and V. V. Wagh, "Health status and personal hygiene among food handlers working at food establishment around a rural teaching hospital in wardha district of Maharashtra, India," Global Journal of Health Science, vol. 2, no. 2, p. 198, 2010.

[8] A. Wendafrash, "Food safety and codex activities in Ethiopia," Foodborne Diseases, pp. 21-26, Environmental Health Department, Addis Ababa, Ethiopia, 2010.

[9] S. Jarmila, V. Babak, H. Martina et al., "Microbial contamination after sanitation of food contact surfaces in dairy and meat processing plants," Czech Journal of Food Sciences, vol. 28, no. 5, pp. 450-461, 2010.

[10] S. Khurana, N. Taneja, R. Thapar, M. Sharma, and N. Malla, "Intestinal bacterial and parasitic infections among food handlers in a tertiary care hospital of north India," Tropical Gastroenterology: Official Journal of the Digestive Diseases Foundation, vol. 29, pp. 207-209, 2008.

[11] G. Andargie, A. Kassu, F. Moges, M. Tiruneh, and K. Huruy, "Prevalence of bacteria and intestinal parasites among foodhandlers in Gondar town, northwest Ethiopia," Journal of Health, Population, and Nutrition, vol. 26, pp. 451-455, 2008.

[12] P. F. Ayeh-Kumi, S. Quarcoo, G. Kwakye-Nuako, J. P. Kretchy, A. Osafo-Kantanka, and S. Mortu, "Prevalence of intestinal parasitic infections among food vendors in accra," The Journal of Tropical Medicine and Parasitology, vol. 32, pp. 1-8, 2009.

[13] D. Marami, K. Hailu, and M. Tolera, "Prevalence and associated factors of intestinal parasitic infections among asymptomatic food handlers working at Haramaya university cafeterias, eastern Ethiopia," Annals of Occupational and Environmental Medicine, vol. 30, no. 1, p. 53, 2018.
[14] E. Scallan, R. M. Hoekstra, F. J. Angulo et al., "Foodborne illness acquired in the United States-major pathogens," Emerging Infectious Diseases, vol. 17, no. 1, pp. 7-15, 2011.

[15] J. Azanaw, M. Gebrehiwot, and H. Dagne, "Factors associated with food safety practices among food handlers: facility-based cross-sectional study," BMC Research Notes, vol. 12, no. 1, p. $683,2019$.

[16] A. G. Tessema, K. A. Gelaye, and D. H. Chercos, "Factors affecting food handling practices among food handlers of Dangila town food and drink establishments, north west Ethiopia," BMC Public Health, vol. 14, no. 1, p. 571, 2014.

[17] M. Kibret and B. Abera, "The sanitary condition of food service establishments and food safety knowledge and practices of food handlers in Bahir Dar town," Ethiopian Journal of Health Sciences, vol. 22, no. 1, pp. 27-35, 2012.

[18] M. M. Zain, "Naing NN owards food sanitation: a preliminary report," Southeast Asian Journal of Tropical Medicine \& Public Health, vol. 33, no. 2, pp. 410-417, 2002.

[19] A. H. Havelaar, A. Cawthorne, F. Angulo et al., "WHO initiative to estimate the global burden of foodborne diseases," The Lancet, vol. 381, p. S59, 2013.

[20] O. Muinde and E. Kuria, "Hygienic and sanitary practices of vendors of street foods in Nairobi, Kenya," African Journal of Food, Agriculture, Nutrition and Development, vol. 5, 2005.

[21] D. Legesse, M. Tilahun, E. Agedew, and D. Haftu, "Food handling practices and associated factors among food handlers in arba minch town public food establishments in Gamo Gofa zone, southern Ethiopia," Epidemiology, vol. 7, no. 302, pp. 2161-2165, 2017.

[22] A. Kumie and K. Zeru, "Sanitary conditions of food establishments in Mekelle town, Tigray, north Ethiopia," The Ethiopian Journal of Health Development, vol. 21, pp. 3-11, 2007.

[23] World Helath Organization, Foodborne Disease: Focus on Health Education, World Helath Organization, Geneva, Switzerland, 2000.

[24] S. O. Nee and N. A. Sani, "Assessment of knowledge, attitudes and practices (KAP) among food handlers at residential colleges and canteen regarding food safety," Sains Malaysiana, vol. 40, no. 4, pp. 403-410, 2011.

[25] R. M. Mickey and S. Greenland, "The impact of confounder selection criteria on effect estimation," American Journal of Epidemiology, vol. 129, no. 1, pp. 125-137, 1989.

[26] L. Daru, A. H. Husodo, S. Iravati, and Z. Shaluhiyah, "Safe food handling knowledge, attitude and practice of food handlers in hospital kitchen," International Journal of Public Health Science, vol. 6, no. 4, pp. 324-330, 2017.

[27] T. Derso, A. Tariku, F. Ambaw, M. Alemenhew, G. A. Biks, and A. Nega, "Socio-demographic factors and availability of piped fountains affect food hygiene practice of food handlers in Bahir Dar town, northwest Ethiopia: a cross-sectional study," BMC Research Notes, vol. 10, no. 1, p. 628, 2017.

[28] L. Sharif, M. M. Obaidat, and M.-R. Al-Dalalah, "Food hygiene knowledge, attitudes and practices of the food handlers in the military hospitals," Food and Nutrition Sciences, vol. 4, no. 3, pp. 245-251, 2013.

[29] S. P. Nyarugwe, A. R. Linnemann, Y. Ren et al., “An intercontinental analysis of food safety culture in view of food safety governance and national values," Food Control, vol. 111, Article ID 107075, 2020.

[30] F. A. Chekol, M. F. Melak, A. K. Belew, and E. G. Zeleke, "Food handling practice and associated factors among food handlers in public food establishments, northwest Ethiopia," BMC Research Notes, vol. 12, p. 20, 2019. 
[31] A. C. Iwu, K. A. Uwakwe, C. B. Duru et al., "Knowledge, attitude and practices of food hygiene among food vendors in Owerri, Imo state, Nigeria," Occupational Diseases and Environmental Medicine, vol. 5, no. 1, pp. 11-25, 2017.

[32] U. Mohlisi Mohd Asmawi, A. Azureen Norehan, K. Salikin et al., "An assessment of knowledge, attitudes and practices in food safety among food handlers engaged in food courts," Current Research in Nutrition and Food Science Journal, vol. 6, no. 2, pp. 346-353, 2018.

[33] M. A. Grassi, E. Osella, and T. Civera, "Analysis of food safety and hygiene in mass catering establishments," Journal of Industie Alimetari, vol. 47, no. 480, pp. 471-478, 2008.

[34] R. Mendagudali, K. Akka, I. Swati, D. Shedole, and N. Bendigeri, "Knowledge, attitude, and practices of food safety among women of Khaza bazar, the urban field practice area of KBN institute of medical sciences, Kalaburagi, Karnataka," International Journal of Medical Science and Public Health, vol. 5, no. 3, pp. 516-520, 2016.

[35] R. Hamid, S. Radu, M. Othman, T. Poh, and C. Lay, "Assessment of knowledge, attitude and practices concerning food safety among restaurant workers in Putrajaya, Malaysia," Journal of Food Science and Quality Management, vol. 32, 2014.

[36] M. Adane, B. Teka, Y. Gismu, G. Halefom, and M. Ademe, "Food hygiene and safety measures among food handlers in street food shops and food establishments of Dessie town, Ethiopia: a community-based cross-sectional study," PLoS One, vol. 13, no. 5, Article ID e0196919, 2018.

[37] A. Kumie, K. Genete, H. Worku, E. Kebede, F. Ayele, and H. Mulugeta, "The sanitary conditions of public food and drink establishments in the district town of Zeway, southern Ethiopia," The Ethiopian Journal of Health Development, vol. 16, pp. 95-104.18, 2002.

[38] B. Michaels, C. Keller, M. Blevins, G. Paoli, T. Ruthman, and E. Todd, "Prevention of food worker transmission of foodborne pathogens: risk assessment and evaluation of effective hygiene intervention strategies," Food Service Technology, vol. 4, no. 1, pp. 31-49, 2004.

[39] I. Tonder, J. F. R. Lues, and M. M. Theron, "The personal and general hygiene practices of food handlers in the delicatessen sections of retail outlets in south Africa," Journal of Environmental Health, vol. 70, no. 4, pp. 33-39, 2007.

[40] M. Angela, "Safe food handlers manual," 2009.

[41] I. Monney, D. Agyei, and W. Owusu, "Hygienic practices among food vendors in educational institutions in Ghana: the case of Konongo,” Foods, vol. 2, no. 3, pp. 282-294, 2013.

[42] M. A. Ansari and Z. Khan, "An evaluation of health education intervention on hygienic status of food handlers in Aligarh-a three-year experience," Journal of Community Medicine \& Health Education, vol. 2, p. 142, 2012. 